\title{
Bilanzierung und Controlling
}

\section{Liebe Leserinnen und Leser,}

Controller agieren nicht in einem institutionenfreien Raum, sondern müssen bei der Gestaltung des Controllinginstrumentariums und der -aufgaben auch die Rahmenbedingungen der Rechnungslegung beachten. In der vorliegenden Ausgabe haben wir ausgewählte Aspekte im Zusammenspiel von Bilanzierung und Controlling zusammengestellt. Dabei stehen in diesem Heft weniger die konkreten Regelungen des HGB oder der IFRS im Vordergrund - zum HGB unter Beachtung der Änderungen des BilMoG wird im Herbst ein Sonderheft erscheinen - sondern die Konsequenzen für das Controlling.

Häufig werden für die Rechnungslegung Informationen benötigt, die letztlich nur vom Controller in seiner Funktion als Informationslieferant bereitgestellt werden können. Gleichzeitig stellt die Rechnungslegung aber Anforderungen an den Umfang und die Häufigkeit der Informationsversorgung. Die Artikel von Harald Wirtz "Die monetäre Bewertung technologiebasierter immaterieller Vermögenswerte" sowie von Stefan Müller und Jens Reinke „Die Abgrenzung von zahlungsmittelgenerierenden Einheiten im Rahmen des Impairment Test aus dem Blickwinkel der internen Unternehmenssteuerung" zielen dabei auf die Anforderungen im Rahmen der Bestimmung von Ertragswerten für einzelne Vermögensgegenstände oder -werte bzw. für Unternehmensteileinheiten. In diesem Zusammenhang möchte ich auch auf den Transferbeitrag von Ann-Sophie Hermens und mir verweisen, in dem wir getreu der Idee dieser Transferarbeit die wesentlichen Ergebnisse wissenschaftlicher Artikel in so genannten A-Journals vorstellen.

Bei den Arbeiten von Matthias J. Rapp „Bilanzorientiertes Risikomanagement in Industrieunternehmen“ und Maximilian Römhild „Bilanzorientierte Kennzahlen als Deduktionsbasis für die wertorientierte Steuerung" wird die Verbindung ebenfalls schon im Aufsatztitel deutlich. Obwohl die Autoren in diesen Beiträgen keine konkreten Rechnungslegungsvorschriften diskutieren, werden dennoch die Zahlen des Rechnungswesens zum Input (und Auslöser) für das Handeln von Controllern.

Neben diesen Themen zeigt Ihnen das aktuelle Heft aber auch die Breite der Herausforderungen eines Controllers, die Ihnen natürlich bestens bekannt ist und für die wir hoffen, eine Anregung zu liefern.

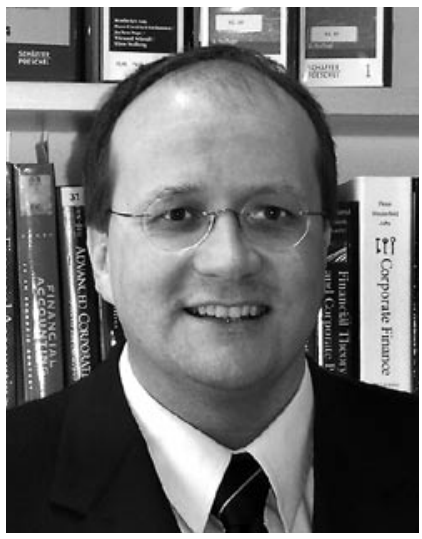

Dirk Hachmeister

Mit besten Grüßen

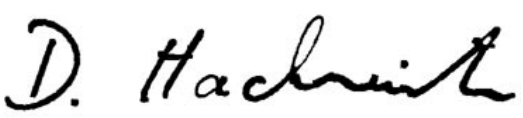

Dirk Hachmeister 